\title{
Bimodal SLD Ice Accretion on a NACA 0012 Airfoil Model
}

\author{
Mark Potapczuk \\ NASA John H. Glenn Research Center, Cleveland, Ohio, 44135 USA \\ Jen-Ching Tsao \\ Ohio Aerospace Institute, Cleveland, Ohio, 44135 USA \\ Laura King-Steen \\ HX5 Sierra, Cleveland, Ohio, 44135 USA \\ Presented at \\ 9th AIAA Atmospheric and Space Environments Conference \\ Denver, CO \\ June 5-9, 2017
}




\section{Outline}

- Objectives

- Approach

- Background

$>$ Facility

$>$ Cloud Conditions

$>$ Model

$>$ Test Procedures

$>$ Test Matrix

- Results

- Concluding Remarks

- Acknowledgements 


\section{Objectives}

1. Document the Ice Shapes Produced using the IRT Bimodal Spray Conditions

2. Compare with Ice Shapes Produced using the Single Nozzle Array (Monomodal) for Equivalent Cloud Conditions

> Use previously produced ice shapes as reference conditions 


\section{Approach}

1. Evaluate the IRT Bimodal Spray Ice Shapes

- At 130, 150, $200 \& 250$ knots

- At $\alpha=0^{\circ}, 4^{\circ}$

2. Compare with Monomodal Spray Ice Shapes at

- 2 Ice Shape Repeatability Conditions

- 2 Ice Shape Condition from Scaling Work 


\section{NASA Icing Research Tunnel}

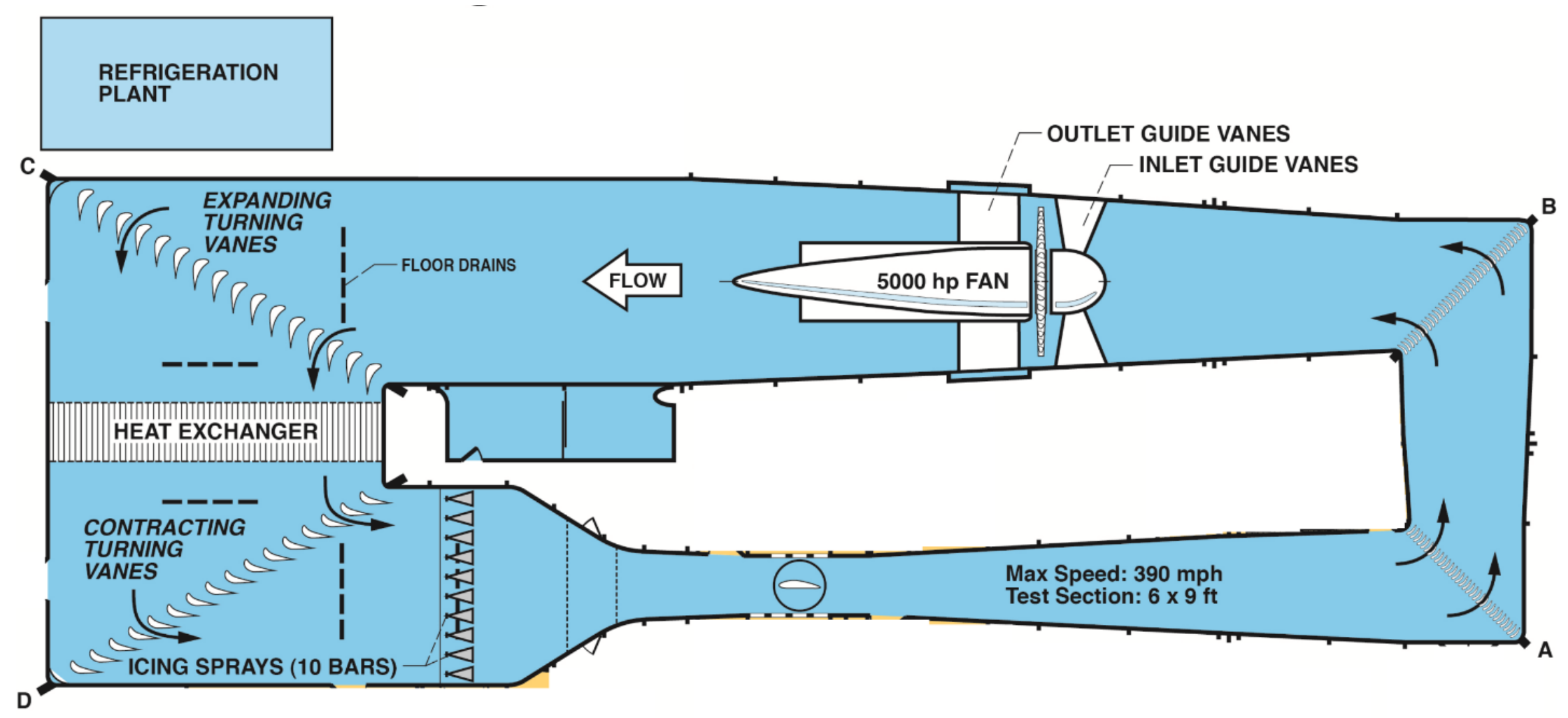

CD-10-83244C 


\section{IRT Bimodal Spray}

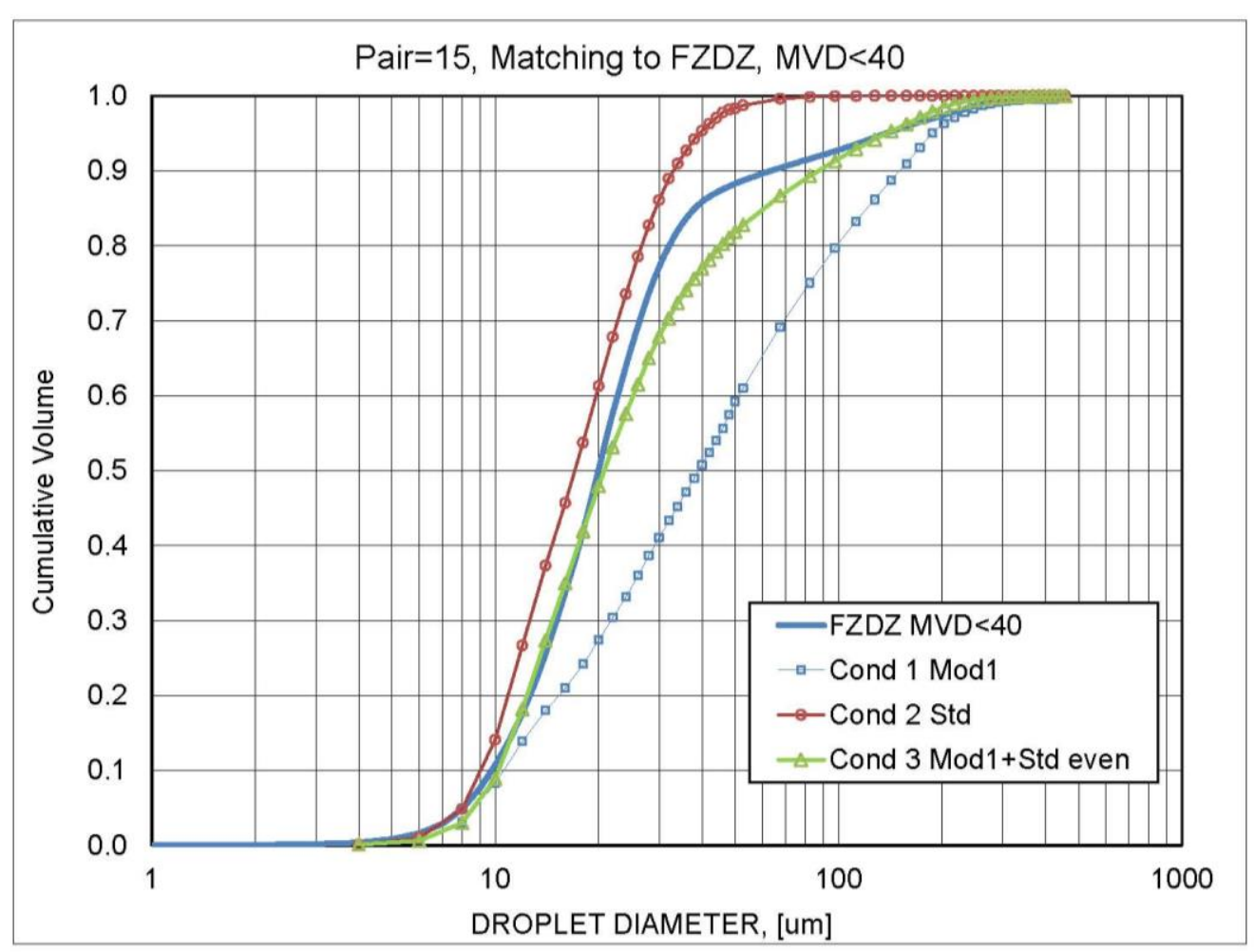

Pair $=15$ psig

- Man1 (Mod1) deltaP = 80 psid

- $\mathrm{MVD}=39.2$ um

- $\min L W C(@ 250 \mathrm{kts})^{*}=0.67 \mathrm{~g} / \mathrm{m}^{3}$

- Man2 (Std) deltaP = 7 psid

- $M V D=17.1$ um

- $\min L W C(@ 250 \mathrm{kts})^{*}=0.78 \mathrm{~g} / \mathrm{m}^{3}$

- Combined:

- $M V D=20.8$ um

- $\operatorname{minLWC}(@ 250 \mathrm{kts}) *=1.45 \mathrm{~g} / \mathrm{m}^{3}$

- How good of a match is it to FZDZ, MVD<40?

- The normalized cumulative LWC in each of the measured bins was within $10 \%$ of what the normalized cumulative LWC is for FZDZ, $M V D<40$ (for each corresponding bin)

* LWC values based on IRT LWC calibration curves 


\section{Selected IRT Mod1 Spray Condition Monomodal Distribution}

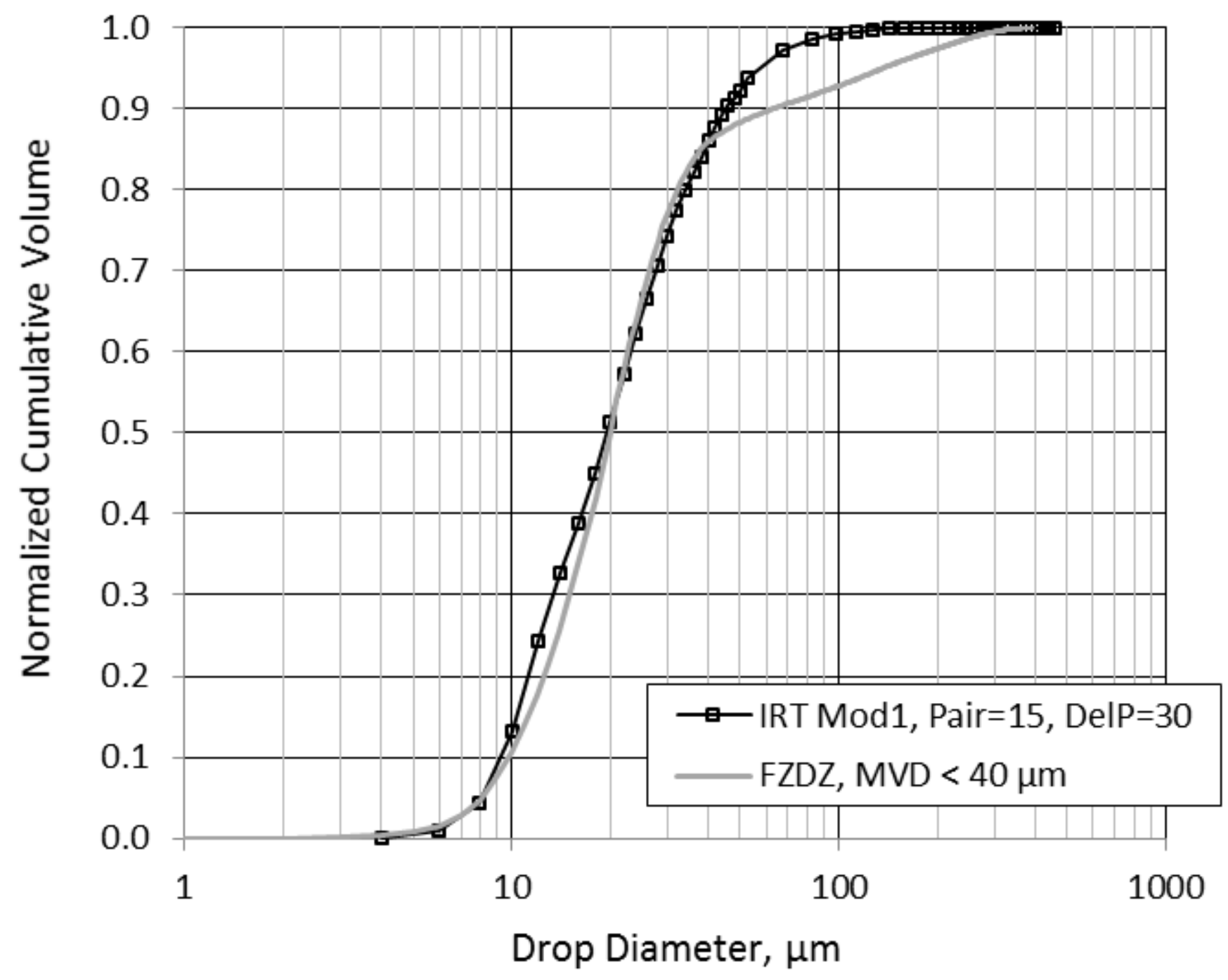




\section{IRT Bimodal \& Monomodal Distributions}

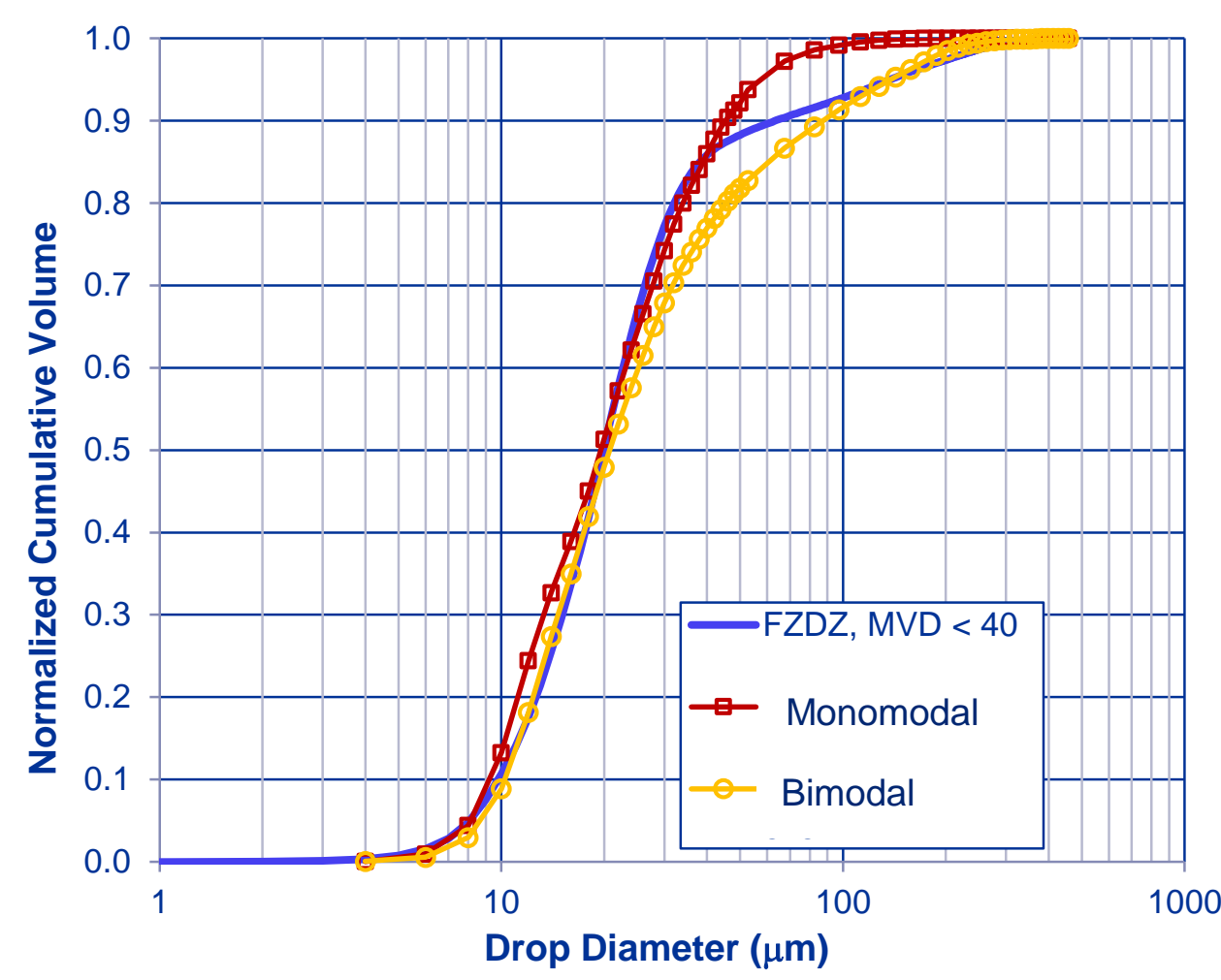

- $\quad F Z D Z, M V D<40$

- FAA App O distribution

- $\quad M V D=20 \mu \mathrm{m}$

_ $\quad$ LWC between 0.29 and $0.44 \mathrm{~g} / \mathrm{m}^{3}$

- Bimodal

- Mod1 + Std nozzles

- $\quad$ Pair = 15 psig

- Mod1 DeltaP $=80$ psid

- $\quad$ Standard DeltaP $=7$ psid

- Combined MVD $=20.8 \mu \mathrm{m}$

- Combined minLWC (@250 kts)=1.45 g/m

- Monomodal

- Mod1 nozzles

- Pair=15 psig

- $\quad$ DelP=30 psid

- $\quad$ MVD=19.3 $\mu \mathrm{m}$

_ $\quad \operatorname{minLWC}(@ 250 \mathrm{kts})=0.37 \mathrm{~g} / \mathrm{m}^{3}$

- Both IRT distributions were measured by spraying only even-numbered spray bars, as is typical for drop-sizing calibrations in Appendix $C$ conditions in order to avoid coincidence error

- LWC values are based on IRT calibration curves 


\section{Test Model}

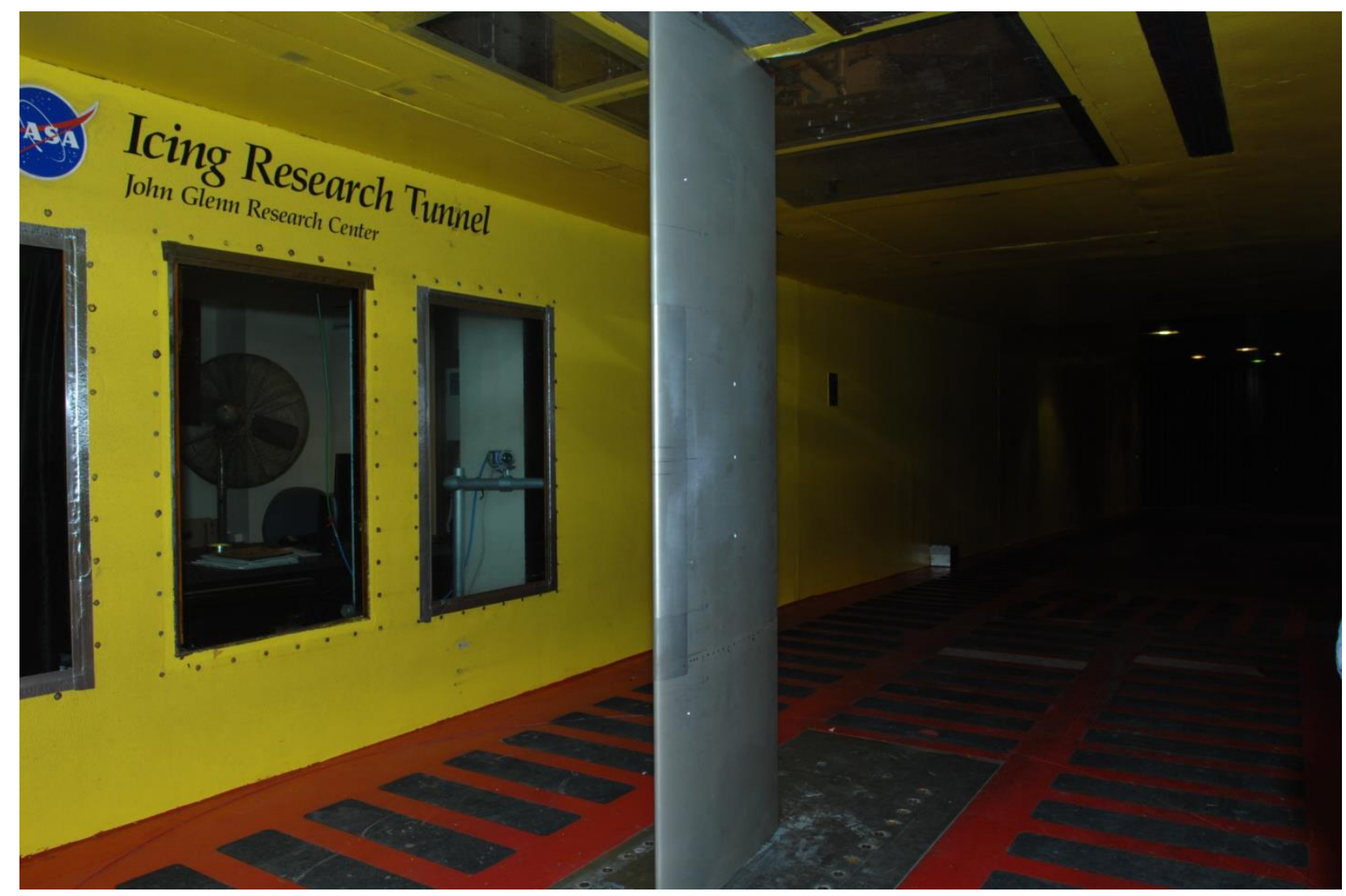

21-in chord NACA 0012 model, full span 


\section{Test Procedures}

- The tunnel temperature and velocity conditions were set.

- The spray bar air and water pressures were set.

- The tunnel was run at the set temperature and velocity conditions and the thermocouples on the model were monitored.

- When the model temperature matched the tunnel static air temperature, the model was considered to be sufficiently cold to initiate the spray.

- The spray was initiated and lasted for the prescribed time for the icing condition of that run.

- After the spray was stopped and the tunnel velocity was reduced to idle conditions, personnel entered the test section and performed the following tasks.

- Photographs of the ice on the model were taken from several pre-set locations around the model.

- A laser scanner system was used to obtain geometric data of the ice shape using the method described by Lee, et al.

- Once the ice shapes were scanned, a 12 inch spanwise section of the ice shape was removed from the surface into a collection tray and weighed in order to obtain the accumulated mass.

- Following the removal of the mass, the model surface was cleaned of all remaining ice and prepared for the next test run. 


\section{Test Matrix}

5 proposed reference conditions

\begin{tabular}{|c|c|c|c|c|c|c|c|c|c|}
\hline \multicolumn{10}{|c|}{ Test Conditions } \\
\hline Case & $\begin{array}{l}\text { Reference } \\
\text { Condition }\end{array}$ & $\alpha$ & $\begin{array}{c}V \\
\text { (kts) }\end{array}$ & $\begin{array}{l}\text { MVD } \\
\text { ( } \mathrm{m})\end{array}$ & $\begin{array}{l}\text { LWC } \\
\left(\mathrm{g} / \mathrm{m}^{3}\right)\end{array}$ & $\begin{array}{c}\mathrm{T}_{\mathrm{t}} \\
\left({ }^{\circ} \mathrm{C}\right)\end{array}$ & $\begin{array}{c}\mathrm{T}_{\mathrm{s}} \\
\left({ }^{\circ} \mathrm{C}\right)\end{array}$ & $\begin{array}{l}\text { Time } \\
\text { (min) }\end{array}$ & $\mathrm{n}_{0}$ \\
\hline $\begin{array}{c}\text { Ice Shape Repeatibility } \\
\text { Run } 3\end{array}$ & 1 & 4 & 200 & 20 & 0.55 & -5.6 & -10.8 & 7 & 0.52 \\
\hline $\begin{array}{c}\text { Ice Shape Repeatibility } \\
\text { Run } 23\end{array}$ & 2 & 4 & 130 & 22 & 1 & -5.6 & -7.8 & 6 & 0.34 \\
\hline 5-15-06/Run 14 & 3 & 0 & 150 & 30 & 1.34 & -12.5 & -15.5 & 5.5 & 0.49 \\
\hline 5-15-06/Run 15 & 4 & 0 & 100 & 30 & 1.75 & -13.5 & -14.8 & 6.7 & 0.5 \\
\hline 3-28-05/Run 6 & 5 & 0 & 250 & 26.8 & 0.56 & -5.2 & -13.4 & 8.5 & 0.46 \\
\hline
\end{tabular}

Note: For scaling, two selected spray clouds are considered 


\section{Test Matrix}

Monomodal and bimodal test conditions based upon scaling of reference conditions.

\begin{tabular}{|c|c|c|c|c|c|c|c|c|c|c|c|c|c|}
\hline Run \# & $\begin{array}{l}\text { Reference } \\
\text { Condition }\end{array}$ & $\alpha$ & $\begin{array}{c}\text { V } \\
\text { (kts) }\end{array}$ & $\begin{array}{l}\text { MVD } \\
(\mathrm{m})\end{array}$ & $\begin{array}{c}\text { LWC } \\
\left(\mathrm{g} / \mathrm{m}^{3}\right)\end{array}$ & $\begin{array}{c}\mathrm{T}_{\mathrm{t}} \\
\left({ }^{\circ} \mathrm{C}\right)\end{array}$ & $\begin{array}{c}\mathrm{T}_{\mathrm{s}} \\
\left({ }^{\circ} \mathrm{C}\right)\end{array}$ & $\begin{array}{l}\text { Time } \\
\text { (min) }\end{array}$ & $\mathrm{n}_{0}$ & $\begin{array}{c}\text { Mod-1 } \\
p_{\text {air }}, \\
\text { psig }\end{array}$ & $\begin{array}{c}\text { Mod-1 } \\
\text { Dp, } \\
\text { psid }\end{array}$ & $\begin{array}{l}\text { Std } \\
p_{\text {air }} \\
\text { psig }\end{array}$ & $\begin{array}{l}\text { Std } \\
\text { Dp, } \\
\text { psid }\end{array}$ \\
\hline AE2716 & $5 . b$ & 0 & 250 & 19.3 & 0.37 & -2.3 & -10.5 & 14 & 0.46 & 15 & 30 & & \\
\hline AE2717 & $2 . b$ & 4 & 130 & 19.3 & 0.55 & -2.8 & -5 & 11.5 & 0.34 & 15 & 30 & & \\
\hline AE2718 & $1 . b$ & 4 & 200 & 19.3 & 0.42 & -3.9 & -9.2 & 9.3 & 0.52 & 15 & 30 & & \\
\hline AE2719 & $2 . a$ & 4 & 130 & 20.8 & 2.15 & -9.9 & -12.1 & 2.9 & 0.34 & 15 & 80 & 15 & 7 \\
\hline AE2720 & $5 . a$ & 0 & 250 & 20.8 & 1.45 & -11.9 & -20.2 & 3.5 & 0.46 & 15 & 80 & 15 & 7 \\
\hline AE2721 & $1 . \mathrm{a}$ & 4 & 200 & 20.8 & 1.64 & -15.2 & -20.5 & 2.3 & 0.52 & 15 & 80 & 15 & 7 \\
\hline AE2738 & $5 . b$ & 0 & 250 & 19.3 & 0.37 & -2.3 & -10.5 & 14 & 0.46 & 15 & 30 & & \\
\hline AE2739 & $2 . b$ & 4 & 130 & 19.3 & 0.55 & -2.8 & -5 & 11.5 & 0.34 & 15 & 30 & & \\
\hline AE2740 & $3 . b$ & 0 & 150 & 19.3 & 0.5 & -4.2 & -7.2 & 17 & 0.49 & 15 & 30 & & \\
\hline AE2741 & $2 . a$ & 4 & 130 & 20.8 & 2.15 & -9.9 & -12.1 & 2.9 & 0.34 & 15 & 80 & 15 & 7 \\
\hline AE2742 & $3 . a$ & 0 & 150 & 20.8 & 1.96 & -14.9 & -17.9 & 4.2 & 0.49 & 15 & 80 & 15 & 7 \\
\hline
\end{tabular}

Note: a - Bimodal spray; b - Monomodal spray 


\section{Olsen Method for Scaling LWC}

1. $C_{s}=C_{r}$

2. $V_{s}=V_{r}$

3. $M V D_{s}=M V D_{r}$

4. Choose a $L W C_{s}$

5. Calculate the scale temperature $T_{s t, s}$ from $n_{0, s}=n_{0, r}$

6. Calculate the scale total temperature, $\mathrm{T}_{t o t, s}$. If $\mathrm{T}_{t o t, s}$ is greater than $-2^{\circ} \mathrm{C}$, repeat steps 4,5 , and 6 with a larger $L W C_{s}$

7. Calculate the scale accretion time from $A_{c, s}=A_{c, r}$, which leads to $t_{s}=\left(L W C_{r} \times t_{r}\right) / L W C_{s}$ 


\section{Sample Photograph and Scan} Test Run \#AE2741
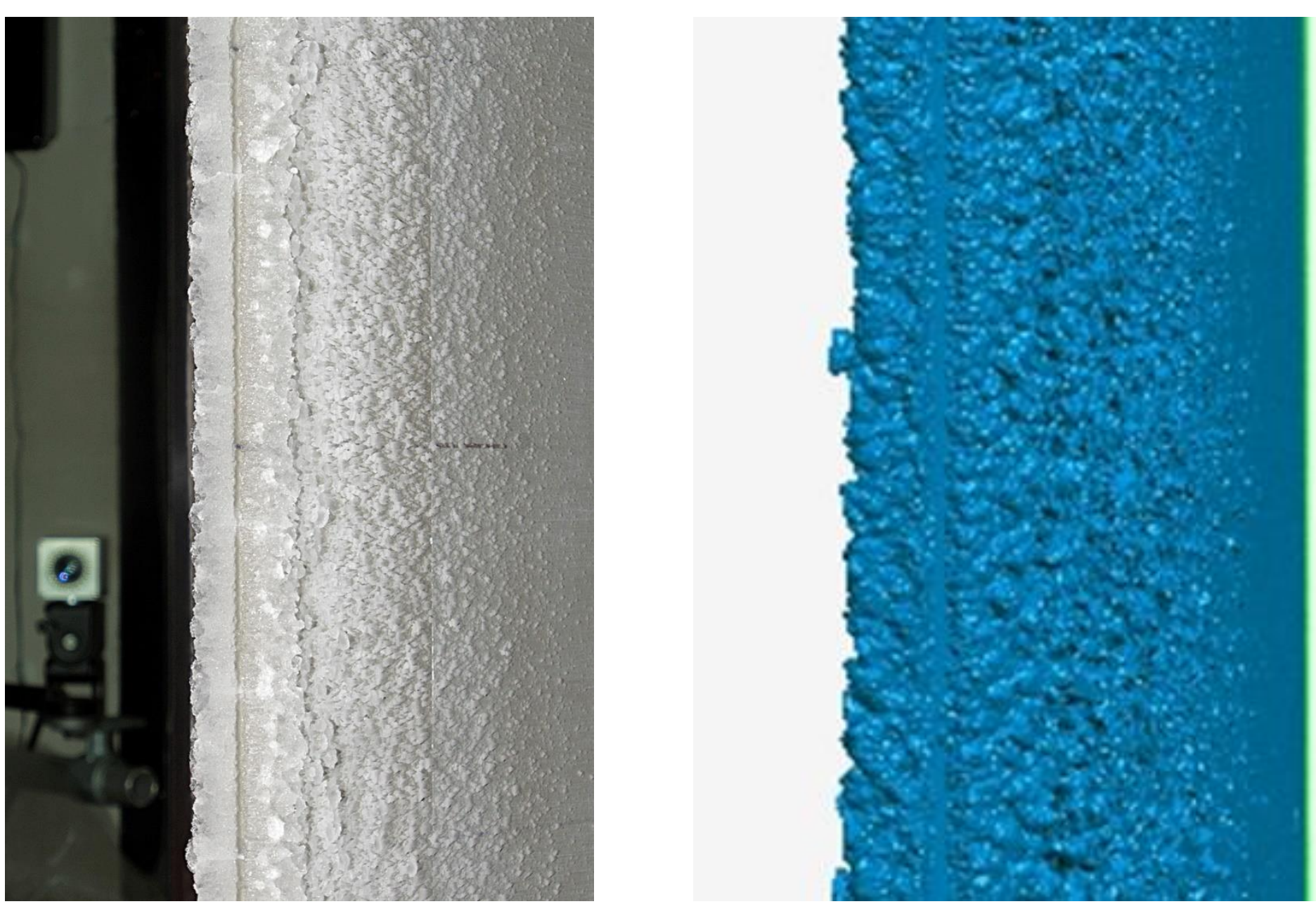


\section{Test Results \\ Quantitative Data}

Mass and volume measurements for the ice shapes resulting from the scaled monomodal and bimodal distribution icing conditions from this test program.

\begin{tabular}{|c|c|c|c|c|c|c|c|c|c|c|c|}
\hline \multicolumn{12}{|c|}{ Test Results } \\
\hline $\begin{array}{l}\text { Reference } \\
\text { Condition }\end{array}$ & $\begin{array}{c}\text { Mass } \\
\text { bimodal } \\
(\mathrm{g}) \\
\end{array}$ & $\begin{array}{c}\text { Mass } \\
\text { monomodal } \\
(\mathrm{g}) \\
\end{array}$ & $\begin{array}{r}\Delta \mathrm{m}_{\mathrm{i}} \\
(\mathrm{g}) \\
\end{array}$ & $\begin{array}{c}\Delta \mathrm{m}_{\mathrm{i}} \\
\% \\
\end{array}$ & $\begin{array}{c}\text { Volume } \\
\text { bimodal } \\
\text { in }^{3} \\
\end{array}$ & $\begin{array}{c}\text { Volume } \\
\text { monomodal } \\
\text { in }^{3} \\
\end{array}$ & $\begin{array}{c}\Delta \text { Vol. } \\
\text { in }^{3} \\
\end{array}$ & $\begin{array}{c}\Delta \text { Vol. } \\
\% \\
\end{array}$ & $\begin{array}{l}\rho_{\text {eff,b }} \\
\mathrm{g} / \mathrm{in}^{3}\end{array}$ & $\begin{array}{l}\rho_{\text {eff, } m} \\
{\mathrm{~g} / \mathrm{in}^{3}}^{3}\end{array}$ & $\begin{array}{c}\Delta \rho_{\text {eff }} \\
\% \\
\end{array}$ \\
\hline 1 & 163.1 & 131.2 & 31.9 & $24 \%$ & 13.67 & 12.39 & 1.28 & $10.3 \%$ & 11.9 & 10.6 & $12.7 \%$ \\
\hline 2 & 151.9 & 137.9 & 14 & $10 \%$ & 14.3 & 11.28 & 3.02 & $26.8 \%$ & 10.6 & 12.2 & $-13.1 \%$ \\
\hline 3 & 207.1 & 188 & 19.1 & $10 \%$ & 18.46 & 15.49 & 2.97 & $19.2 \%$ & 11.2 & 12.1 & $-7.6 \%$ \\
\hline 5 & 228.5 & 157.8 & 70.7 & $45 \%$ & 19.52 & 13.56 & 5.96 & $44.0 \%$ & 11.7 & 11.6 & $0.6 \%$ \\
\hline
\end{tabular}

Note: Density of ice at $0^{\circ} \mathrm{C}$ is $0.9167 \mathrm{~g} / \mathrm{cm}^{3}=15.02 \mathrm{~g} / \mathrm{in}^{3}$ 


\section{Ice Shape Comparisons to Reference Shapes}

Reference Condition 1, V= 200 knots

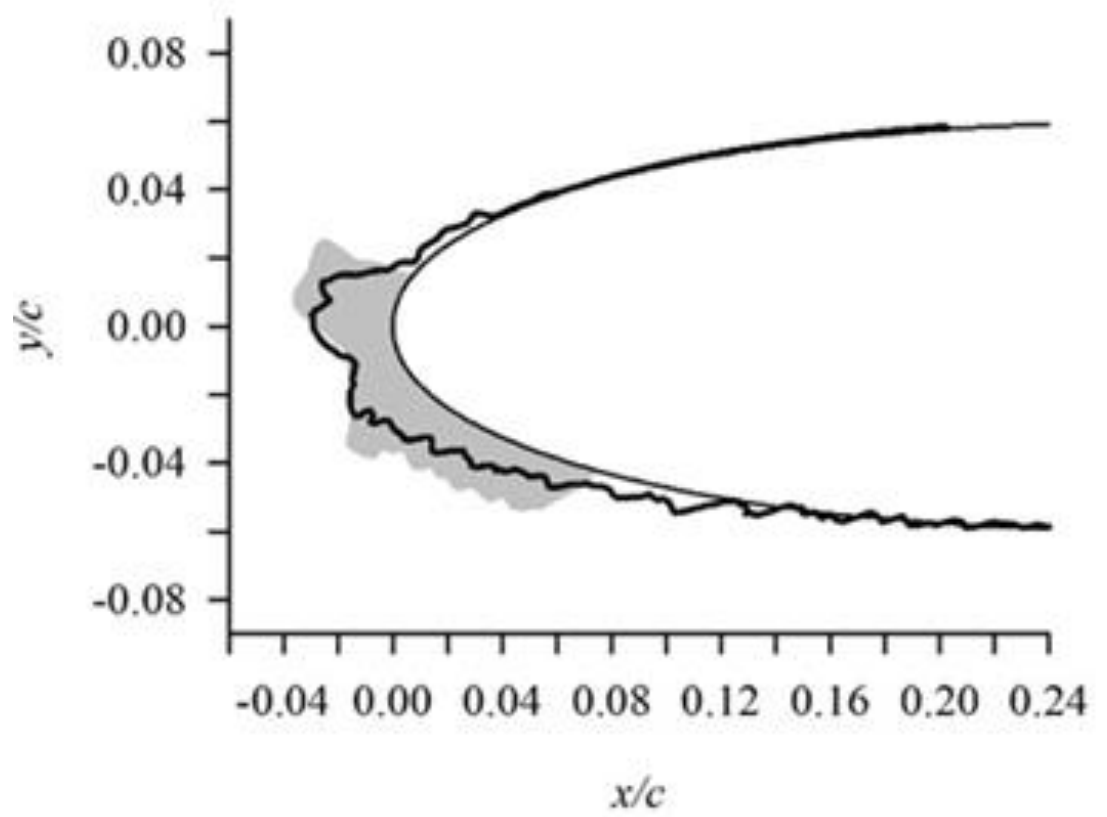

2/18/14 AE2 $102 \mathrm{CL}$, Ref 1
6/01/16 Run 6 CL, Scale 1a

$\mathrm{MVD}_{1}=20 \mu \mathrm{m}, \mathrm{LWC}_{1}=0.55 \mathrm{~g} / \mathrm{m}^{3}, \mathrm{t}_{1}=7 \mathrm{~min}$

$\mathrm{MVD}_{1 \mathrm{a}}=20.8 \mu \mathrm{m}, \mathrm{LWC}_{1 \mathrm{a}}=1.64 \mathrm{~g} / \mathrm{m}^{3}, \mathrm{t}_{1 \mathrm{a}}=2.3 \mathrm{~min}$

\section{Bimodal Distribution (a)}

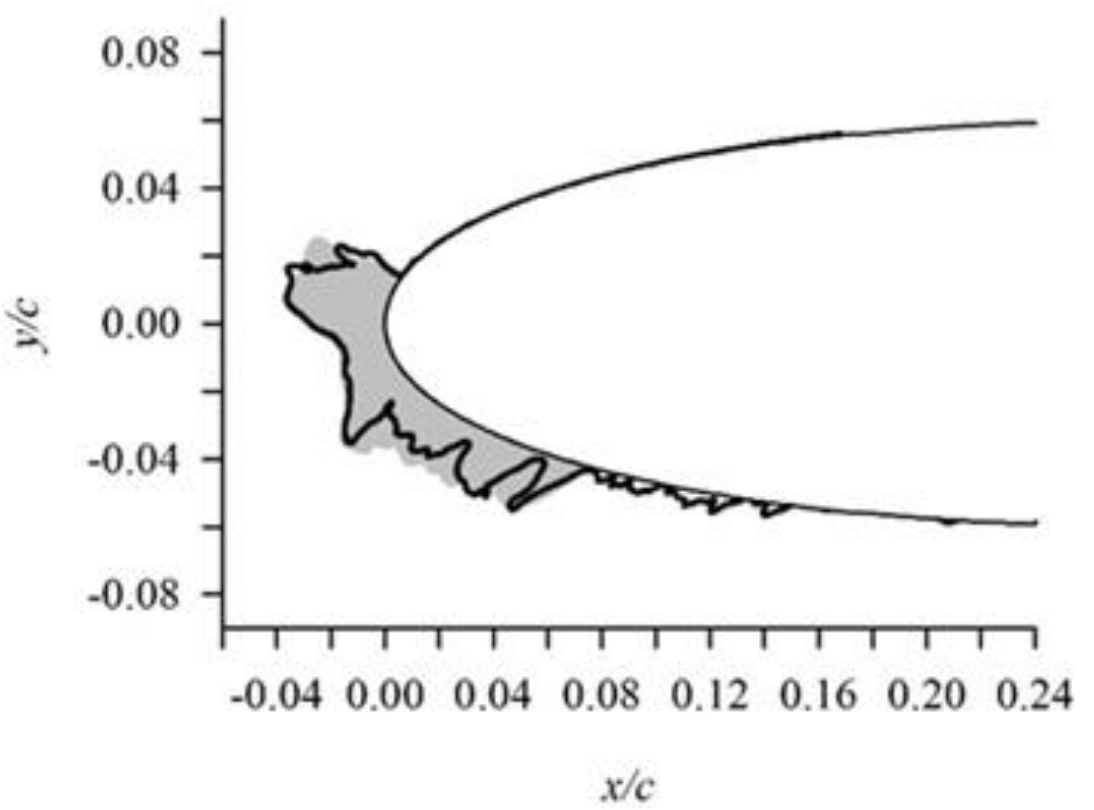

$\square$ 2/18/14 AE2102 CL, Ref 1
$-6 / 01 / 16$ Run 3 CL, Scale 1b

$\mathrm{MVD}_{1}=20 \mu \mathrm{m}, \mathrm{LWC}_{1}=0.55 \mathrm{~g} / \mathrm{m}^{3}, \mathrm{t}_{1}=7 \mathrm{~min}$ $\mathrm{MVD}_{1 \mathrm{~b}}=19.3 \mu \mathrm{m}, \mathrm{LWC}_{1 \mathrm{~b}}=0.42 \mathrm{~g} / \mathrm{m}^{3}, \mathrm{t}_{1 \mathrm{~b}}=9.3 \mathrm{~min}$

Monomodal Distribution (b) 


\section{Ice Shape Comparisons to Reference Shapes}

Reference Condition 2, V = 130 knots

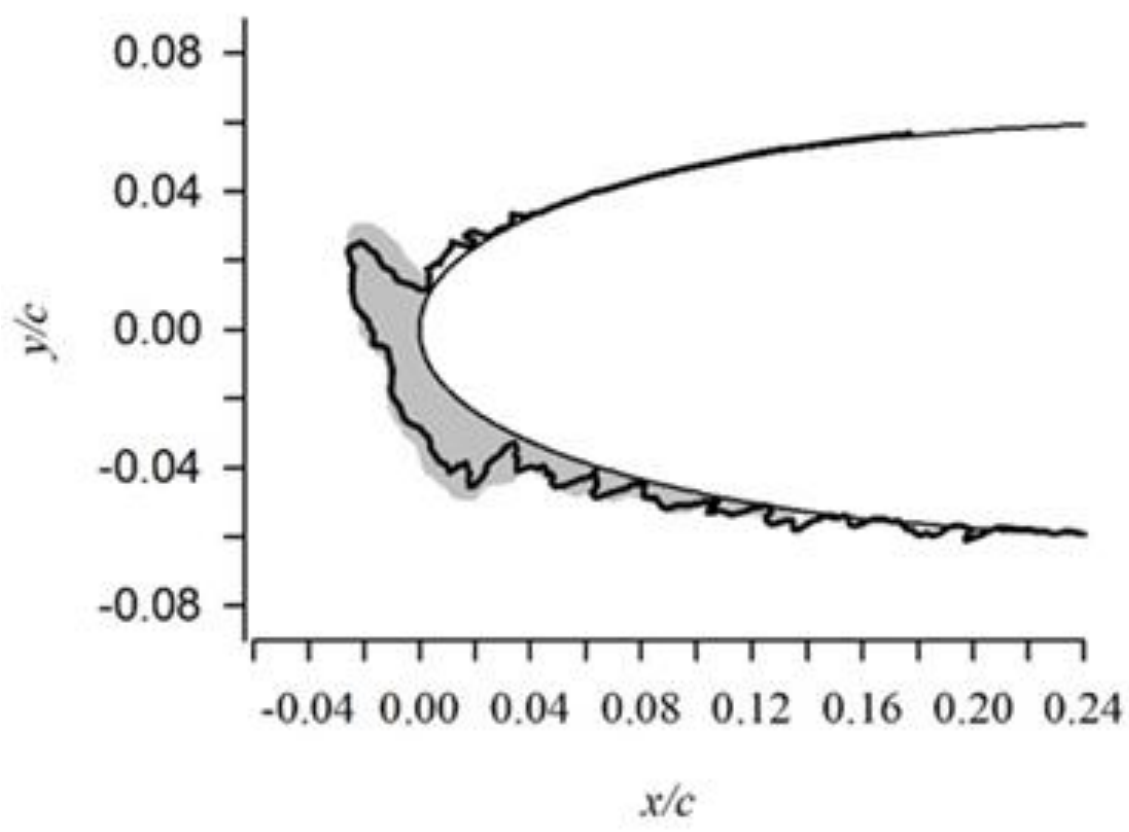

2/19/14 AE2112 CL, Ref 2

- 6/01/16 Run 4 CL, Scale 2a

$\mathrm{MVD}_{2}=22 \mu \mathrm{m}, \mathrm{LWC}_{2}=1.00 \mathrm{~g} / \mathrm{m}^{3}, \mathrm{t}_{2}=6 \mathrm{~min}$

$\mathrm{MVD}_{2 \mathrm{a}}=20.8 \mu \mathrm{m}, \mathrm{LWC}_{2 \mathrm{a}}=2.15 \mathrm{~g} / \mathrm{m}^{3}, \mathrm{t}_{2 \mathrm{a}}=2.9 \mathrm{~min}$

Bimodal Distribution (a)

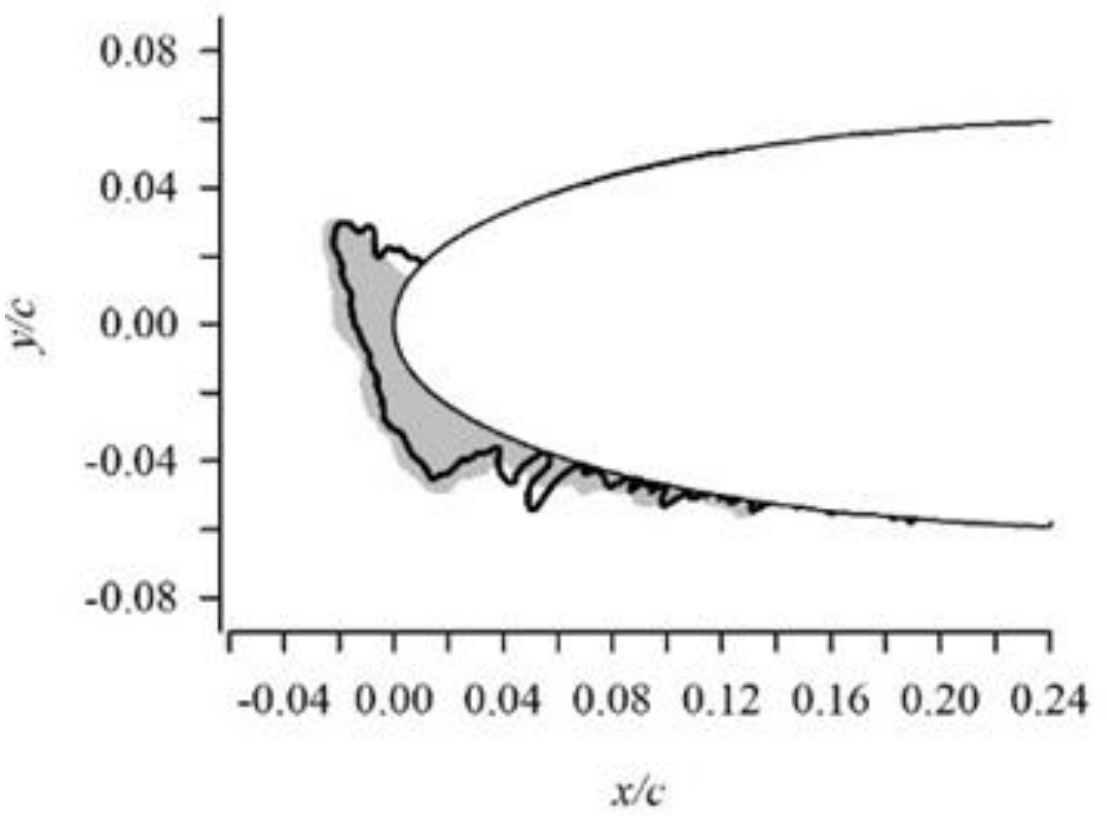

$\square$ 2/19/14 AE2112 CL, Ref 2

- 6/01/16 Run 2 CL, Scale 2b

$\mathrm{MVD}_{2}=22 \mu \mathrm{m}, \mathrm{LWC}_{2}=1.00 \mathrm{~g} / \mathrm{m}^{3}, \mathrm{t}_{2}=6 \mathrm{~min}$ $\mathrm{MVD}_{2 \mathrm{~b}}=19.3 \mu \mathrm{m}, \mathrm{LWC}_{2 \mathrm{~b}}=0.55 \mathrm{~g} / \mathrm{m}^{3}, \mathrm{t}_{2 \mathrm{~b}}=11.5 \mathrm{~min}$

Monomodal Distribution (b) 


\section{Ice Shape Comparisons to Reference Shapes}

Reference Condition 3, V = 150 knots

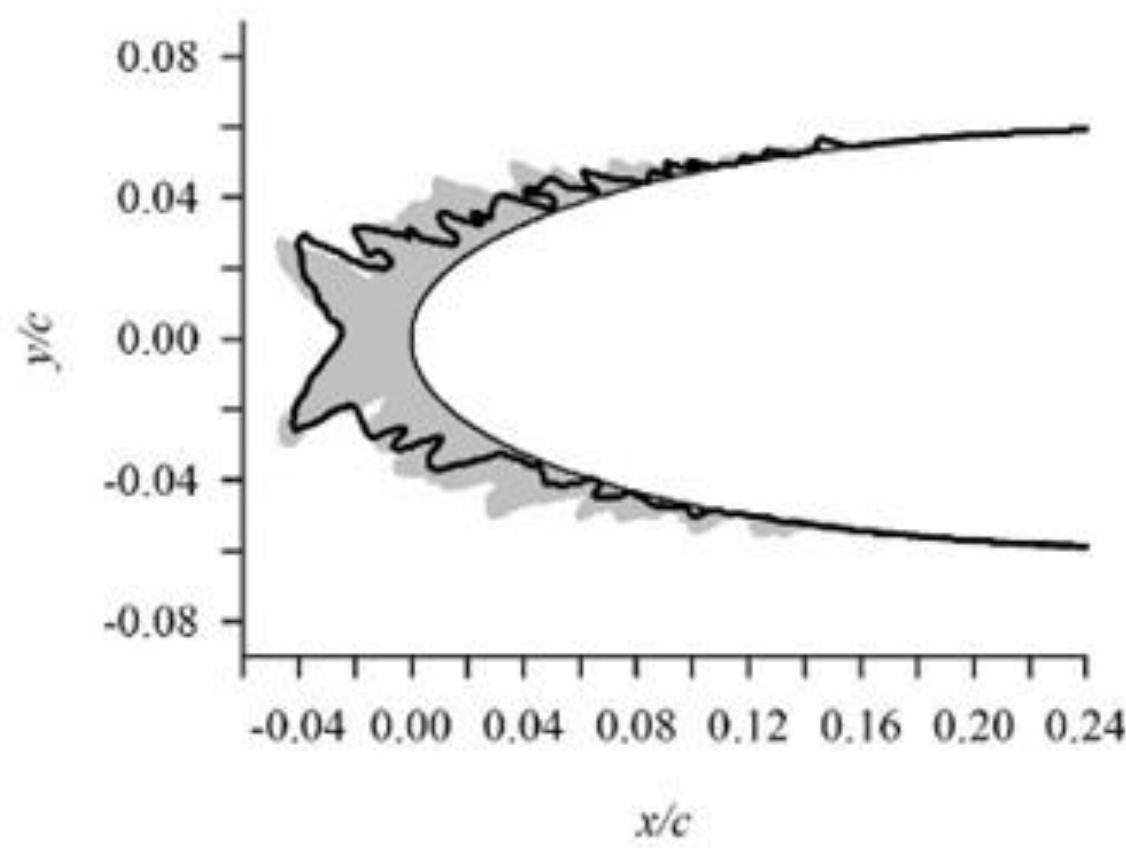

5/15/06 Run 14 Cl., Ref 3

- 8/17/16 Run 5 CL, Scale 3a

$\mathrm{MVD}_{3}=30 \mu \mathrm{m}, \mathrm{LWC}_{3}=1.34 \mathrm{~g} / \mathrm{m}^{3}, \mathrm{t}_{3}=5.5 \mathrm{~min}$ $\mathrm{MVD}_{3 \mathrm{a}}=20.8 \mu \mathrm{m}, \mathrm{LWC}_{3 \mathrm{a}}=1.96 \mathrm{~g} / \mathrm{m}^{3}, \mathrm{t}_{3 \mathrm{a}}=4.2 \mathrm{~min}$

Bimodal Distribution (a)

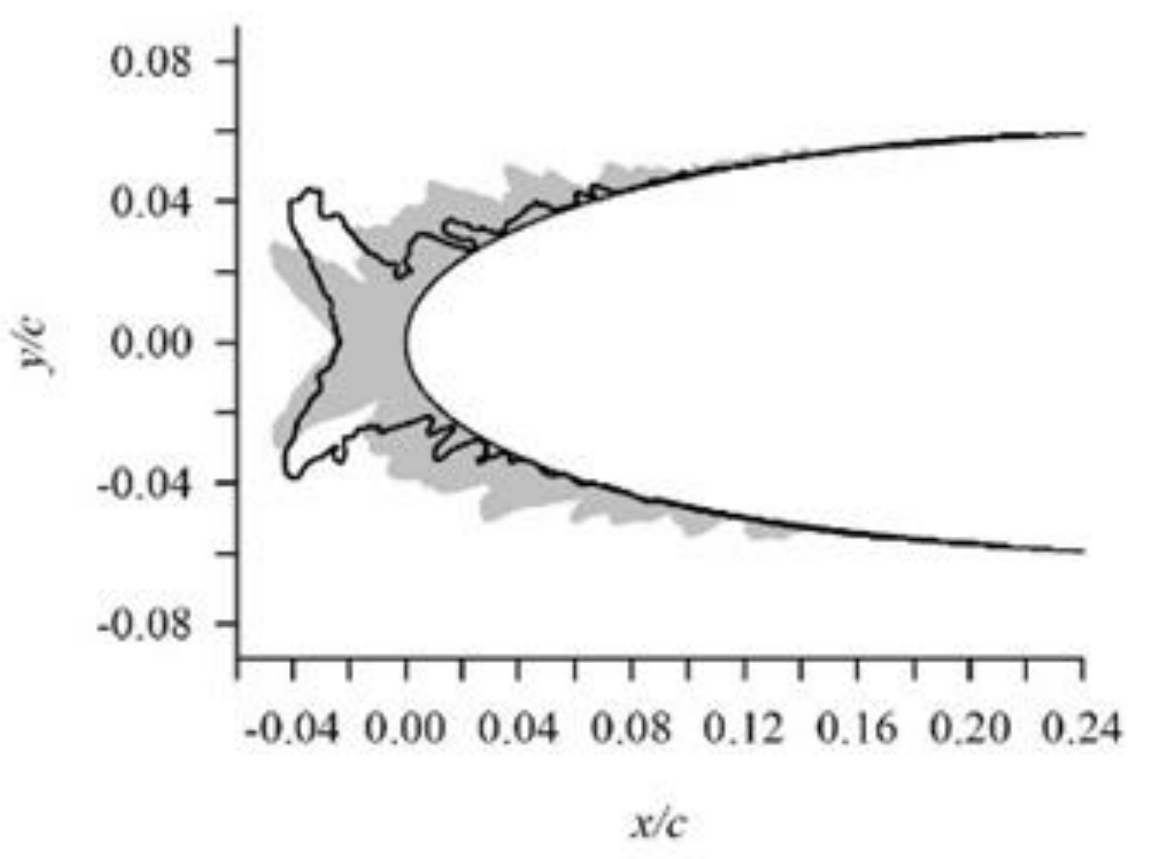

$\square$ 5/15/06 Run 14 CL, Ref 3 8/17/16 Run $3 \mathrm{CL}$, Scale 3b

$\mathrm{MVD}_{3}=30 \mu \mathrm{m}, \mathrm{LWC}_{3}=1.34 \mathrm{~g} / \mathrm{m}^{3}, \mathrm{t}_{3}=5.5 \mathrm{~min}$ $\mathrm{MVD}_{3 \mathrm{~b}}=19.3 \mu \mathrm{m}, \mathrm{LWC}_{3 \mathrm{~b}}=0.5 \mathrm{~g} / \mathrm{m}^{3}, \mathrm{t}_{3 \mathrm{~b}}=17 \mathrm{~min}$

Monomodal Distribution (b) 


\section{Ice Shape Comparisons to Reference Shapes}

Reference Condition 5, V = 250 knots

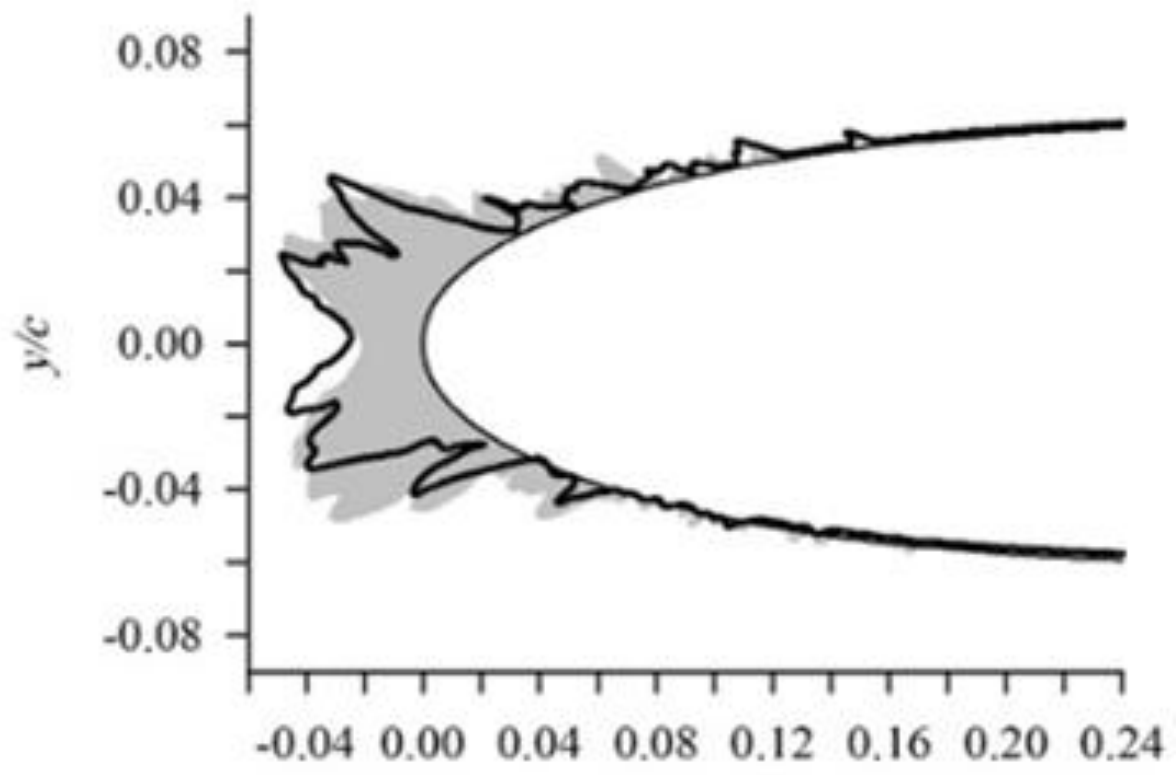

$x / c$

3/28/05 Run 6 CL, Ref 5

- 6/01/16 Run $5 \mathrm{CL}$, Scale 5a

$\mathrm{MVD}_{5}=26.8 \mu \mathrm{m}, \mathrm{LWC}_{5}=0.56 \mathrm{~g} / \mathrm{m}^{3}, \mathrm{t}_{5}=8.5 \mathrm{~min}$ $\mathrm{MVD}_{5 \mathrm{a}}=20.8 \mu \mathrm{m}, \mathrm{LWC}_{5 \mathrm{a}}=1.45 \mathrm{~g} / \mathrm{m}^{3}, \mathrm{t}_{5 \mathrm{a}}=3.5 \mathrm{~min}$

Bimodal Distribution (a)

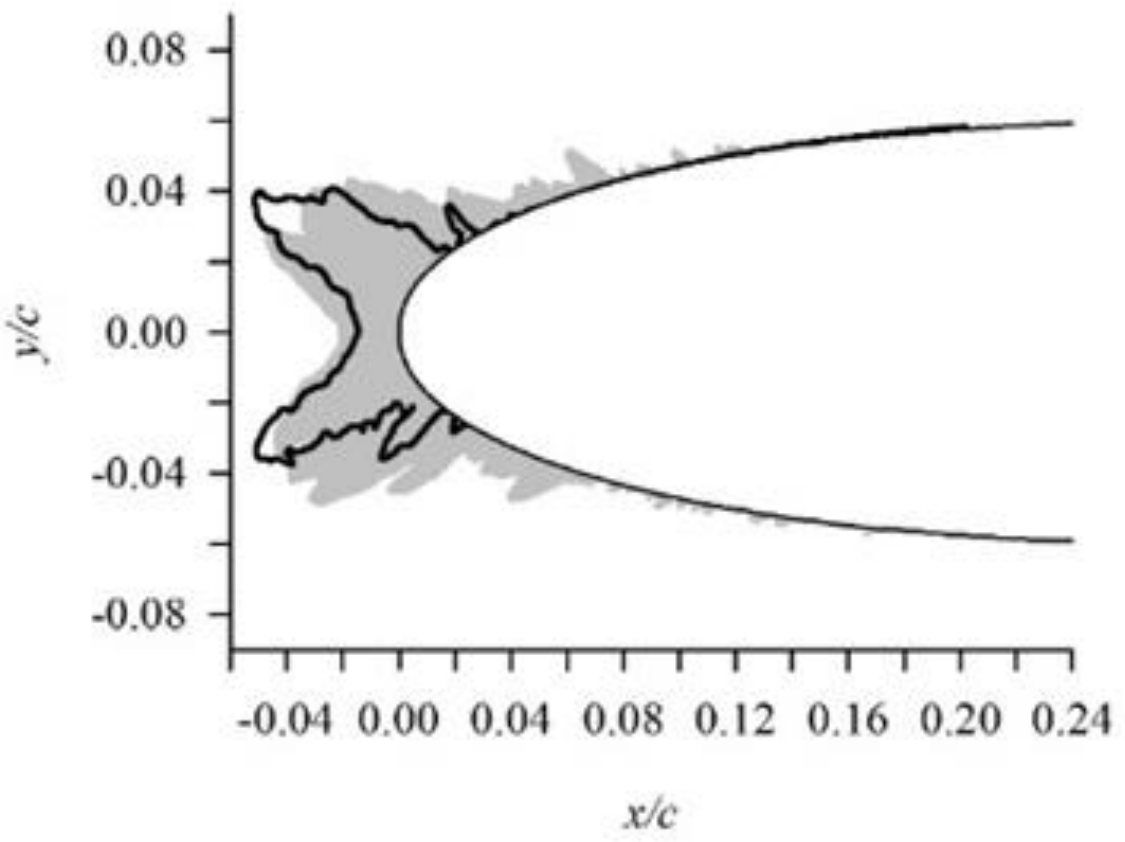

$\mathrm{MVD}_{5}=26.8 \mu \mathrm{m}, \mathrm{LWC}_{5}=0.56 \mathrm{~g} / \mathrm{m}^{3}, \mathrm{t}_{5}=8.5 \mathrm{~min}$ $M \mathrm{MVD}_{5 \mathrm{~b}}=19.3 \mu \mathrm{m}, \mathrm{LWC}_{5 \mathrm{~b}}=0.37 \mathrm{~g} / \mathrm{m}^{3}, \mathrm{t}_{5 \mathrm{~b}}=14 \mathrm{~min}$

Monomodal Distribution (b) 


\section{Bimodal Cloud Effects on Ice Shapes}
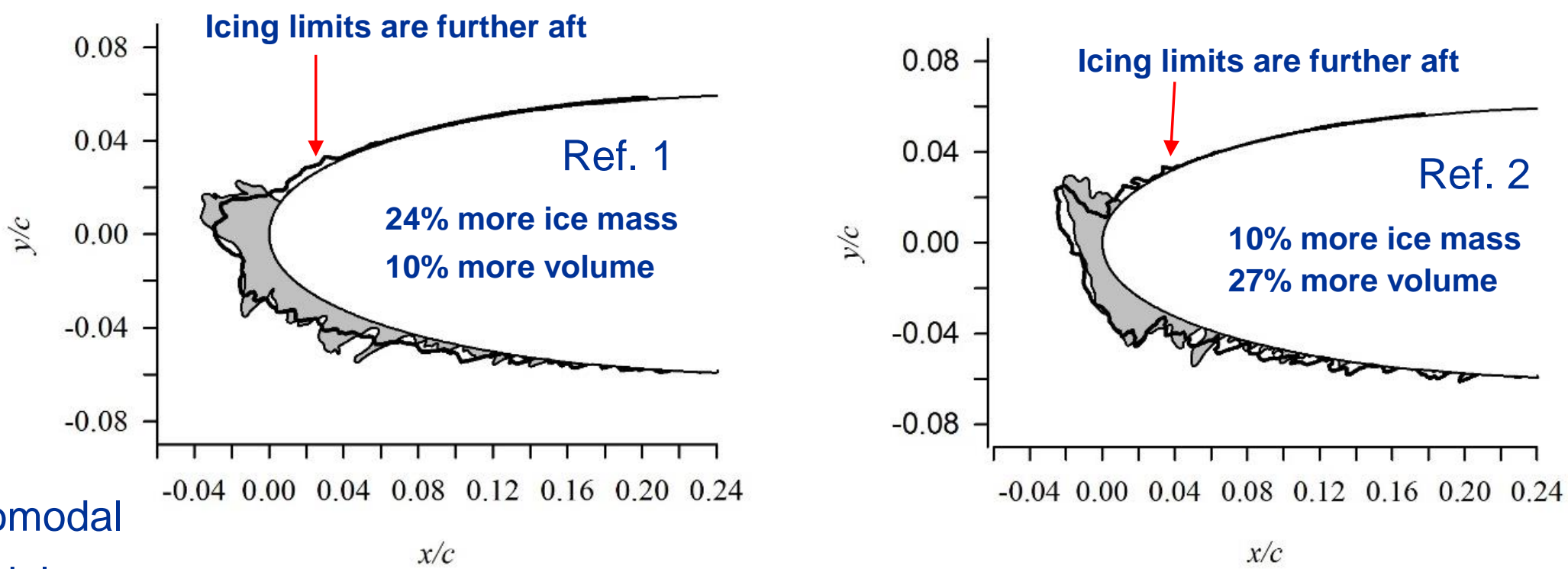

bimodal
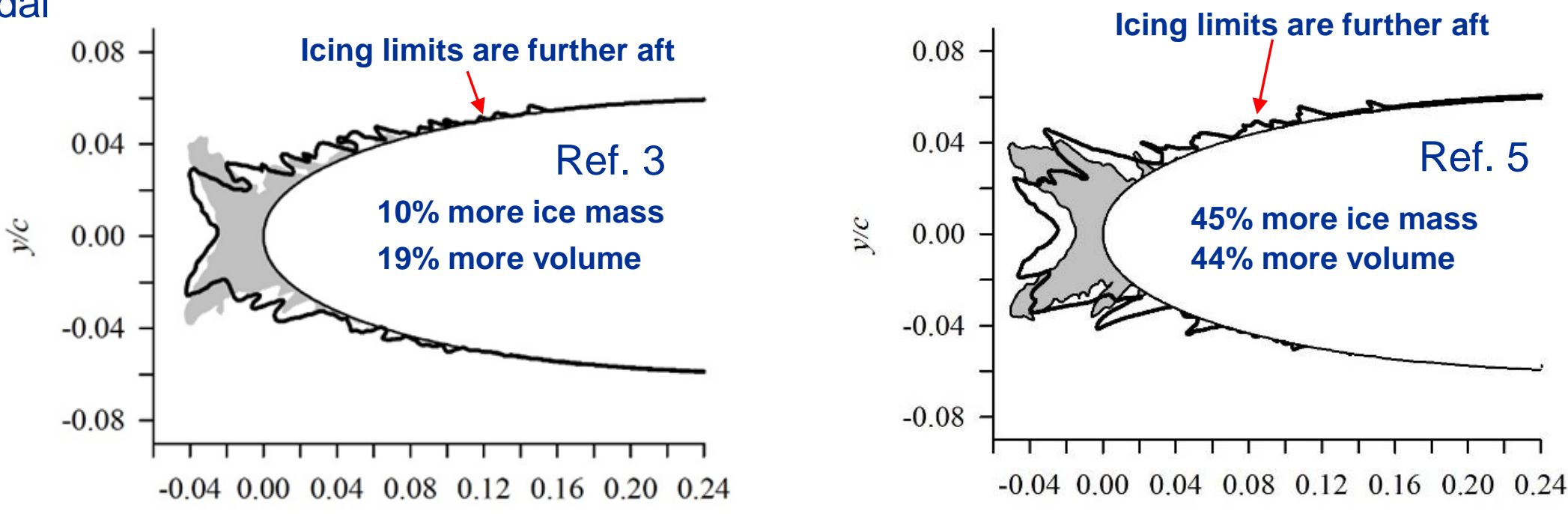

$x / c$

$x / c$ 


\section{Ice Shape Repeatability}

Reference Condition 2
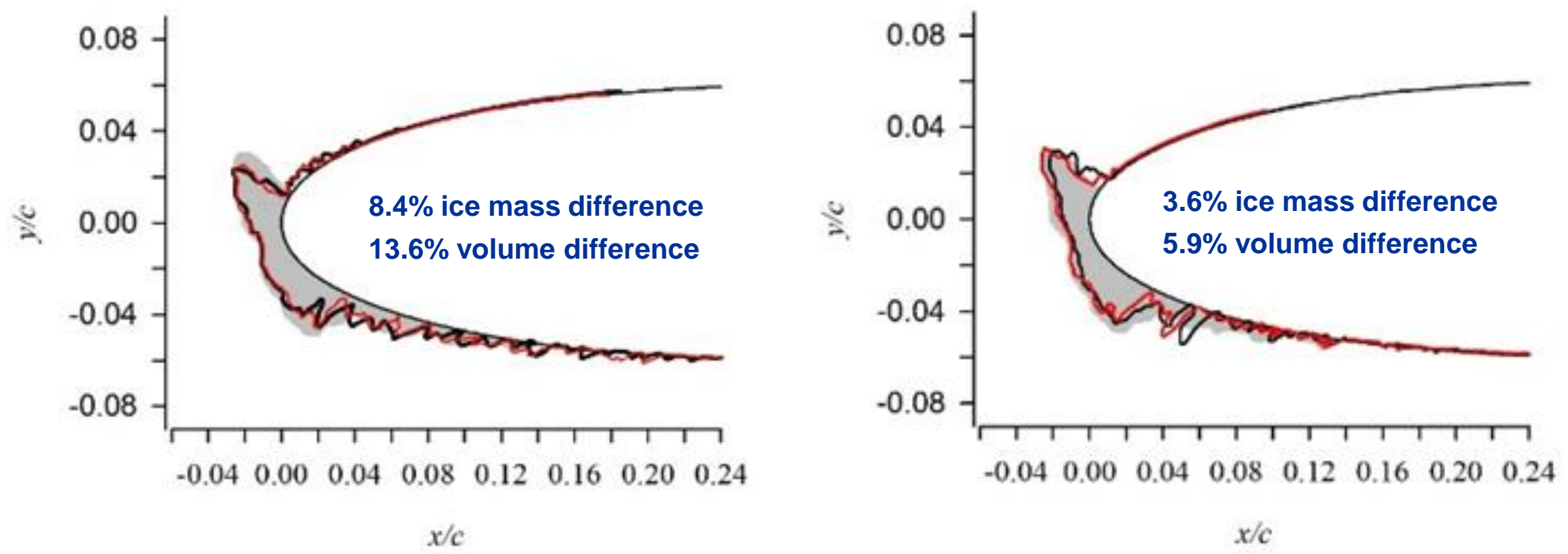

2/19/14 AF2112 CI., Ref 2

- 8/17/16 Run 4 CL,Scale 2a (repeat)

2/19/14 AE2112 CL, Ref 2

6/01/16 Run 2 CL, Scale 2b

6/1/16 Run 4 CL, Scale 2a

- 8/17/16 Run 2 CL, Scale 2 b (repeat)

Bimodal Distribution (a)

Monomodal Distribution (b) 
Normalized Ice Mass Difference

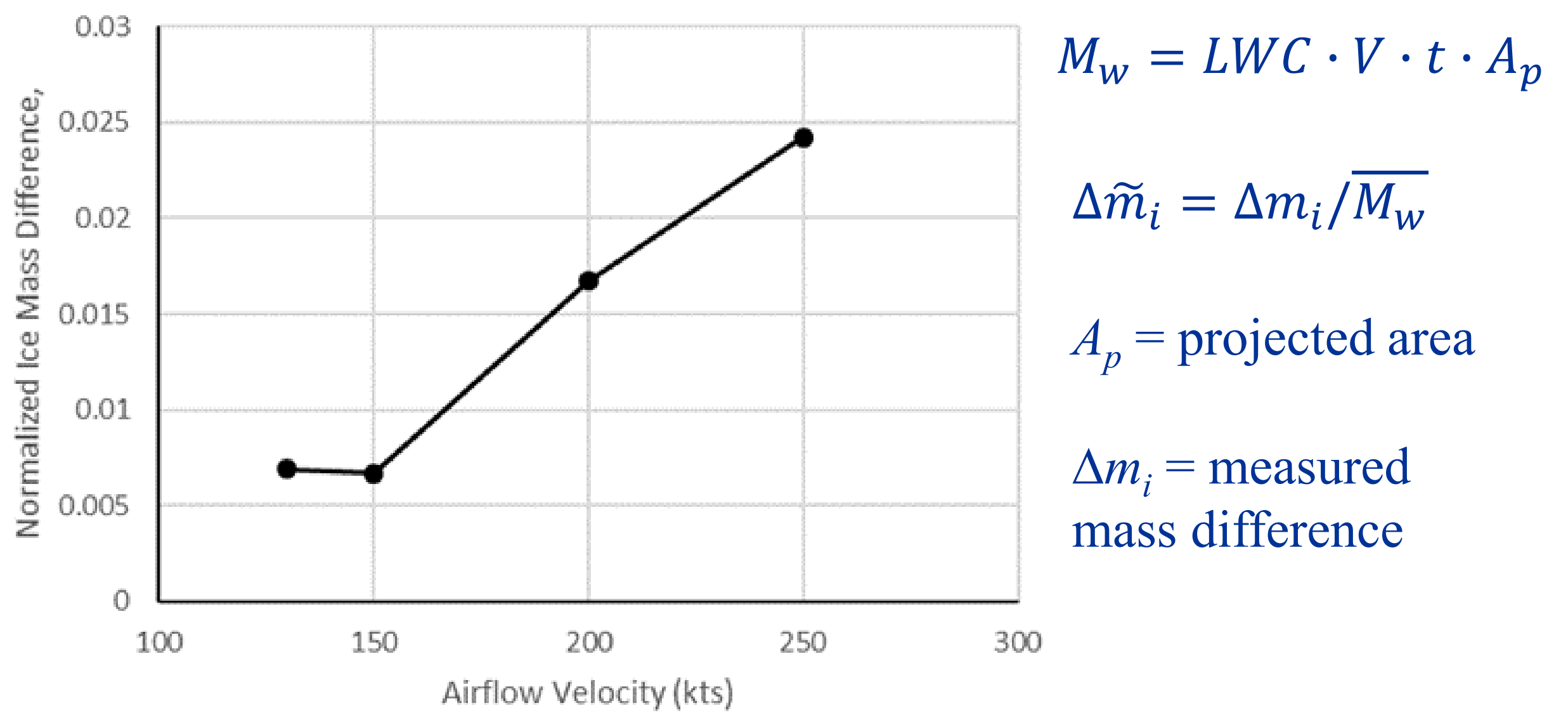




\section{Concluding Remarks}

- Bimodal spray ice shapes were created based upon the simultaneous spray process of Steen and Ide

- Test conditions, using monomodal and bimodal spray distributions, were developed for comparison to previously tested and recorded conditions

- For conditions that were the nominally the same, using the Olsen scaling method, the bimodal ice shapes:

$\checkmark$ Had a larger mass

$\checkmark$ Had a greater volume

$\checkmark$ Had icing limits further aft on the airfoil

- The ice mass difference seemed to increase with increasing velocity

- These differences seemed to be somewhat larger than repeatability

- More Evaluation Tests Recommended 


\section{Acknowledgements}

The authors would like to thank Quentin Schwinn and Jordan Salkin of Alcyon Technical Services (ATS) JV, LLC for their invaluable support in ice shape scanning and post-processing.

The authors would also like to thank the IRT staff for their support in advocating for this work and during the test campaign.

This work was supported through the Aeronautics Evaluation and Test Capabilities (AETC) Project. 